\title{
The GNAM monitoring system and the OHP histogram presenter for ATLAS
}

\author{
P. Adragna*†, G. Crosetti ${ }^{\ddagger}$, M. Della Pietra ${ }^{\S}$, A. Dotti*, R. Ferrari ${ }^{\Uparrow}$, G. Gaudio $₫$, \\ C. Roda*, D. Salvatore ${ }^{\ddagger}$, F. Sarri*, W. Vandelli ${ }^{\Uparrow}$, P. F. Zema ${ }^{\ddagger}$ \\ *University and INFN Pisa, Pisa, Italy \\ ${ }^{\dagger}$ now at University of Siena, Siena, Italy \\ $\ddagger$ University of Calabria and INFN Cosenza, Cosenza, Italy \\ $\S$ University and INFN Napoli, Napoli, Italy \\ ฯUniversity and INFN Pavia, Pavia, Italy
}

\begin{abstract}
ATLAS[1] is one of the four experiments under construction along the Large Hadron Collider at CERN. During the 2004 Combined Test Beam, the GNAM monitoring system and the OHP histogram presenter were widely used to assess both the hardware setup and the data quality. GNAM is a modular framework where detector specific code can be easily plugged in to obtain online low-level monitoring applications. It is based on the monitoring tools provided by the ATLAS Trigger and Data Acquisition (TDAQ) software[2]. OHP is a histogram presenter, capable to perform both as a configurable display and as a browser. From OHP, requests to execute simple interactive operations (such as reset, rebin or update) on histograms, can be sent to GNAM.
\end{abstract}

\section{INTRODUCTION}

ATLAS is one the four experiments being assembled at CERN, along the Large Hadron Collider (LHC), that will provide proton-proton collisions with an energy of $14 \mathrm{TeV}$ in the center-of-mass reference system.

During the last few years much efforts were put in carrying out test beam sessions in order to assess the performances of each ATLAS detector. Different monitoring systems were developed for these data takings, to ease the setup of correct running conditions and the assessment of data quality.

The experience gained, with such programs, afterwards led to the development of a common framework (GNAM), which could be used by every detector, to monitor hardware functionalities, and of a common graphic user interface for histogram displaying (OHP), which could be used by all the monitoring programs.

\section{A. ATLAS}

The layout of the ATLAS detector is driven by the magnet configuration: a solenoidal field around the inner cavity and a toroidal field for the muon spectrometer. Track measurement in the inner region is obtained combining in the Inner Detector (ID) different techniques: high resolution silicon pixels (Pixel detector) and strips (SemiConductor Tracking, SCT) for the region closer to the interaction point and straw-tubes (Transition Radiation Tracking, TRT) in the outer region. A highly granular Liquid Argon electromagnetic sampling calorimeter (LArg) followed by a sampling scintillator/Lead hadronic section (TileCal) cover the central region. The high radiation resistant Liquid Argon technology is also used for the hadronic calorimeter for the endcap and forward regions. The calorimeter system is surrounded by the muon spectrometer which is built exploiting different technologies in order to obtain in each rapidity region high precision tracking (Monitor Drift Tubes, MDT and Cathode Strip Chambers, CSC), fast response (Resistive Plate Chambers, RPC and Thin Gap Chambers) while maintaining a high enough radiation resistance.

The all ATLAS detector consists of about 140 millions of electronic channels. LHC will provide collisions with a frequency of $40 \mathrm{MHz}$, and the output of the first level trigger will be about $10^{5} \mathrm{~Hz}$. This frequency will be further reduced by the higher trigger levels, and finally some hundreds of events will be selected and stored every second.

Considering the huge number of channels and the high event rate, a monitoring system is an essential tool to assess the status of the hardware and the quality of the data while they are being acquired.

\section{B. ATLAS TDAQ}

In ATLAS there are many levels of data-flow[3]. Data are acquired by the front-end electronics (FE), located next to the detectors, and are collected, step by step, until the full event is assembled. DAQ specifications do not impose any fixed structure for the data sent out by the FE, but from the next level on the format is fixed and independent of the data source. Furthermore, any event or fragment must consist of an integer number of 32 bit words.

Part of the FE is devoted to the first trigger level. If a signal is generated, all the front-end boards send data to the Read Out Drivers (ROD), where they are formatted in a detector specific way and enclosed between the standard ATLAS header and trailer. Each ROD is connected to one Read Out Buffer (ROB), which in turn is connected to one ROD only, from which it receives data. A ROB specific header will be added as well.

Up to eighteen ROBs can be part of a Read Out System (ROS), which concatenates the fragments received by all ROBs and, once more, adds some information at the beginning of the 
resultant ROS fragment. At this stage data are examined again for the second level trigger.

Upon receival of a second level trigger signal, all the ROS fragments are joined together and the full event is built, with a process that is very similar to the concatenation of the ROB fragments.

Finally, the event is analyzed and the definitive decision is taken, whether it should be discarded or stored on disk.

Two on-line data monitoring frameworks are foreseen in ATLAS. The low-level monitoring will run mainly at the ROD and ROS levels and will be devoted to check the hardware conditions (dead/noisy channels, electronic mappings, ...) as well at the data quality (synchronizations among different detectors, ...). The high-level monitoring will run mainly on the full events, and will check physics related quantities, such as momentum spectra, invariant masses and so on.

We developed GNAM as a tool for low-level monitoring and $\mathrm{OHP}$ as a general purpose presenter for on-line monitoring histograms.

\section{Monitoring services}

The ATLAS Trigger and Data Acquisition group (TDAQ) offers a number of services to implement on-line applications. Some of them are specially devoted to monitoring purposes, and both GNAM and OHP fully exploit them.

The Information Service (IS) is used for archiving and sharing information among the various DAQ applications; IS is typically used to route information such as run conditions, beam parameters, log messages, however IS is general enough to address any kind of information.

Indeed, the Online Histograms Service (OHS) uses the IS to manage histograms, providing a transient storage between histogram producers and displays. The OHS allows also the routing of commands. Infact any application can issue a command related to a particular histogram, the OHS takes care of sending the command to the appropriate histogram provider.

The Event Monitoring Service (EMS) provides data samplers at the ROD, ROS and SFI levels, that run in parallel with the main data taking. Monitoring applications can request events to the EMS, at a fixed rate or at the maximum possible rate; the request will be satisfied up to a rate that does not affect the data taking performance.

\section{GNAM MONITORING APPLICATION}

An on-line monitoring system should fulfill the following requirements:

1) its architecture should be detector independent: processing a fragment should rely only on its structure, and not on its content;

2) it should be modular, in order to keep common actions separated from detector specific ones; common actions are, for example, interacting with the TDAQ system, unpacking the sampled fragments (taking the relevant parts out of it) and managing the histograms (having them displayed, respond to commands); detector specific actions include data decoding

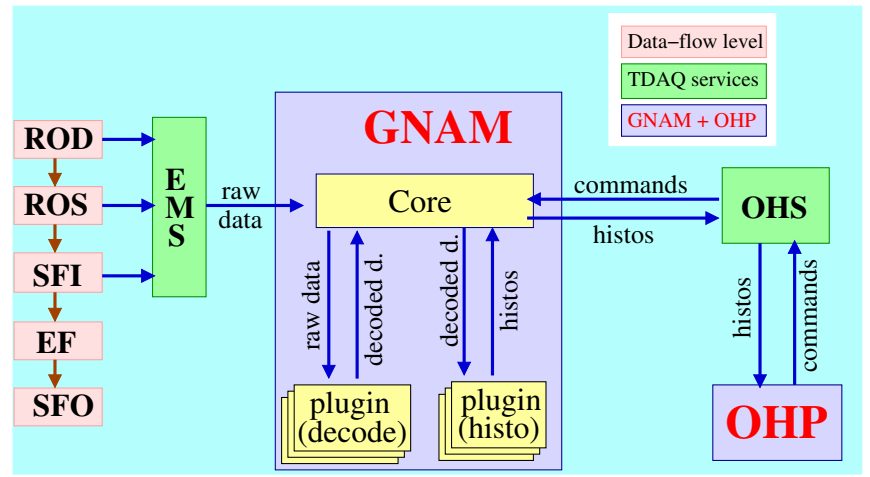

Fig. 1. Schema of the GNAM and OHP monitoring chain

(understanding the physics contents) and analyzing, histogram booking and filling.

3) the monitoring system should be able to run automatically, controlled by the DAQ system;

4) furthermore, monitoring information (mainly histograms) should be collected and stored whenever needed, whether or not there is actually anybody checking it on-line.

\section{A. Monitoring chain}

The schema of our monitoring chain is shown in fig. 1 .

GNAM is divided into two parts: the Core and the detector plugins. The Core handles the common actions, while detector specific code is implemented in the plugins. The Core parses, at run time, a configuration file, where the plugins to be loaded and the initialization parameters are specified.

The Core is responsible for asking event fragments to the EMS and unpacking them, ending up with a list of ROD fragments; it cannot go further because there is no standard format for the data inside the ROD. It is up to each plugin, within its decoding routine, to find the relevant RODs and decode them.

The decoded data are then collected back by the Core and provided to the histogramming plugin, where they will are analyzed and used to fill histograms.

The aim of having all the data decoded before calling any histogramming routine is to allow correlation histograms. Indeed, as every histogramming function can access all the decoded data, it is easy to study the correlation of different detectors, with separate plugins, without duplicating the effort of decoding the raw data.

The Core is also responsible for publishing the histograms on the OHS. Histogram publication may be triggered either when a predefined number of events $\left(N_{m} a x\right)$ have been acquired or when a timeout has expired. Both $N_{\max }$ and the timeout can be set via command line parameters when invoking GNAM.

Histograms are not published all together in a single shot; rather, GNAM only publishes a bunch of histograms at a time, until a fixed number (20000) of bins have been transmitted to OHS. We use this method in order to scatter the dead times along the run and to optimize the network occupancy. 
Finally, the OHP catches the histograms from OHS and displays them. It gives to the user the opportunity of sending back commands to GNAM. At present, three commands are supported:

- Reset, for clearing the content of a histogram;

- Rebin, for changing the number and/or width of the bins;

- Publish, for refreshing it immediately on the OHS.

Reset and Rebin may be addressed to one histogram only or to all the histograms managed by GNAM.

\section{B. GNAM architecture}

The ATLAS DAQ system was implemented as a Finite State Machine. A very simplified schema would comprehend these states: INITIAL, when the applications have just started; CONFIGURED, when every initialization task has been successfully completed; RUNNING, when the data are being acquired. Furthermore, there is the possibility of pausing a run without interrupting it (PAUSE).

In order to have GNAM able to run synchronously with the DAQ, we implemented it as a FSM as well. The transition from INITIAL to CONFIGURED involves two series of actions:

- the configuration file is parsed, all the necessary plugins are loaded and their initialization functions are called;

- the Core registers itself on the specified DAQ partition and opens a connection to the specified event sampler and OHS server.

Upon receiving a START command, the Core switches its state to RUNNING and starts to request events to the sampler. As soon as an event is received, it is unpacked, decoded and analyzed, and histograms are filled, as previously described.

For debugging purposes GNAM has been designed to be also controllable interactively. This allows to easily start and stop the monitoring processes without interfering with the data taking.

\section{C. file_sampler}

The file_sampler is a tool that was developed for debugging purposes. This application reads data files produced at any data flow level and feeds a standard EMS of a specified partition. This application also allows to unpack and write out events up to any specified data flow level (SFI, ROS, ROD).

Using the file_sampler, it is possible to run GNAM, or any other TDAQ application, even when no real data taking is running.

The file_sampler, especially in conjunction with the possibility of controlling GNAM interactively, proved to be very useful when developing or debugging, either the Core or any detector plugin.

\section{Histogram technology}

We chose ROOT[4] as histogramming package because it is a powerful and well known tool for physics analysis. We enhanced ROOT histogram capabilities adding three boolean members: IsPublished, IsFilled and IsResetAtSOR.

IsPublished and IsFilled can be used to selectively activate part of the histograms. The aim is to let detector plugins to

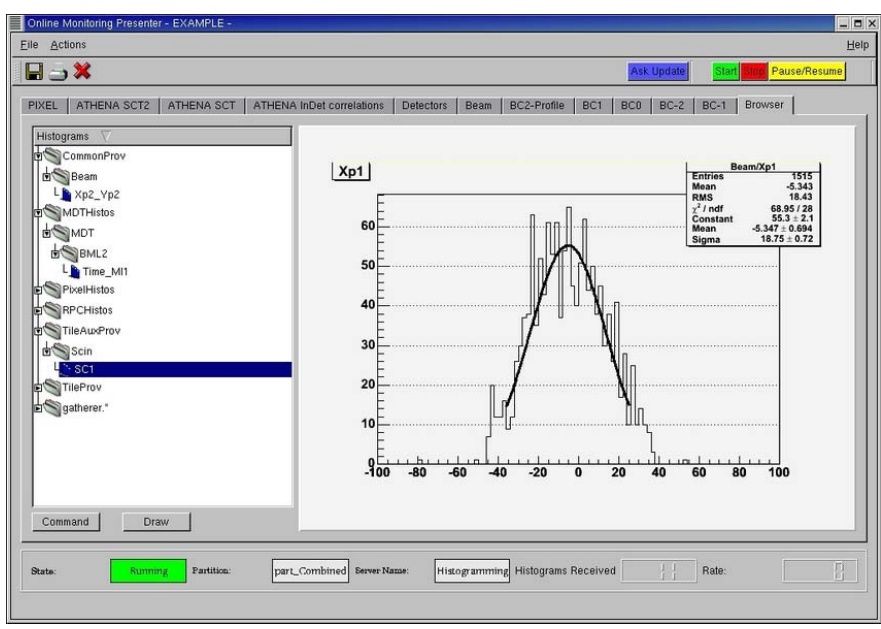

Fig. 2. OHP: a snapshot of the Online Histogram Presenter in browser mode

declare lots of histograms and use them only if necessary. This mechanism allows to investigate a problem in detail switching on the publishing or filling of the appropriate histograms only when necessary, thus saving resource usage and network load when possible.

Histograms are reset by default when a new run starts, but it may sometimes be desirable to collect statistics through many runs. IsResetAtSOR can be used with this intent.

\section{Online Histogram Presenter}

The requirements that he OHP had to fulfill are the following:

1) it should be able to browse the whole content of the OHS, as well as showing a small set of selected histograms;

2) it should be able to send commands back to the histogram producers;

3) it should allow the user to perform standard operations on the histograms, such as fitting or zooming;

4) it should not be tightly linked to GNAM, or to any other histogram producer, since it should be able to manage histograms provided by any producer;

5) it should be able to manage reference histograms.

\section{A. OHP features}

OHP (fig.2) receives histograms from the OHS, this makes the presenter application independent of the histogram producer. OHP can operate in two different modes: it can browse the OHS and it can show a configurable set of histograms in a series of tabs. The two modes allow both the detector experts and the standard shifters to have needed functionalities within the same application. Histograms and tabs to be displayed are specified in a text configuration file that may also be modified from a GUI which may be started from the OHP. The configuration file allows also to specify graphical parameters of the histograms and the list of reference histograms. In the browser mode the histograms the list of received histograms is shown as a folder structure which is built parsing the name of 
the histogram. As already mentioned, the underlying histogram technology is ROOT thus all the standard operations such as fitting and zooming may be executed using the context menus. The interactive commands, such as reset or rebin, to be sent to the histogram provider applications, are accessible using preconfigured buttons and panels of the OHP. The routing of the commands is managed by the OHS as previously discussed.

\section{Combined Test BeAm 2004}

The ATLAS Combined Test Beam[5] (CTB) was held during last year, from May to November; two slices of ATLAS, one in the central and one in the forward region, were exposed to a beam in the North Area of the SPS accelerator. GNAM was used at the very beginning of the data taking in order to set the correct beam conditions and during the whole data taking by various detectors (Pixel, TileCal, MDT and RPC). The fact that the same monitoring system was used by various detectors proved that GNAM is a flexible enough framework.

GNAM was used along the whole test beam to inspect detector status, to find faulty states, to check calibrations and so on. At the end of the CTB, when most detectors where running smoothly, OHP was able to manage more than 1000 histograms, produced by six different GNAM processes running on two low-cost PCs.

\section{COMMISSIONING}

In a few months, all ATLAS detectors will be undergoing the final commissioning phase, with a big load of pre- and post-installation tests.

Some detectors are already using GNAM and OHP during test runs (Tile, MDT and RPC) and others expressed interest in using them: Pixel, TRT, LArg and TGC.

The commissioning phase however will be more complex than the CTB from many points of view, so both GNAM and OHP need to be upgraded.

For what concerns GNAM, the main effort is going in the design and implementation of the interaction with official databases. The interaction with the condition database will allow to trace the detector setup and geometry and to archive the monitoring results, such as histograms.

Although the OHP presenter proved to be a very useful tool during the combined test beam, this experience also highlighted its weak points. The main problem was found in the mechanism of histogram receival which was implemented according to the push mechanism. If the number of produced histograms is not large, this mechanism allows to easily receive up-to-date histograms; however, as the number of histograms increases, the network is heavily loaded receiving histograms that are not even looked at. Secondly, in order to improve the time performances, the presenter will be constructed as a multi-thread application. For these reasons the OHP presenter is being redesigned, in order to be well suited to the increased complexity of both the commissioning and the ATLAS data taking phases.

\section{CONCLUSions}

The GNAM on-line monitoring system and the OHP histogram presenter are fully integrated in the ATLAS TDAQ framework.

GNAM can be used to monitor hardware status and to assess data quality while data are being acquired. OHP is a general purpose histogram presenter, able to retrieve histograms from the OHS, independently of the producer, and display them.

Both of them were used for the first time during the Combined Test Beam 2004; they proved to be light, flexible and extensible. They were very useful to the shift crew, to monitor the status of many detectors at a time, and to the detector experts, who could easily investigate many kinds of problems.

GNAM and OHP are already being used for the ATLAS commissioning, and we are enhancing both of them for the increasing needs relatead to such a complex phase.

Our final goal is to use the experience gained at the CTB and during the comissioning to upgrade both our tools to be suitable for the ATLAS data taking.

\section{ACKNOWLEDGMENT}

The authors would like to thank the developers of the user libraries used during the CTB: I. Boiko, D. Dedovich and K. Nikolaev from the MDT subdetector; D. Dobos, G. Gagliardi, P. Morettini and P. Risso from the Pixel subdetector. We would like also to thank M. Caprini and S. Kolos from ATLAS TDAQ group and the test beam coordinator B. Di Girolamo. A special thank goes to E. Pasqualucci for his support in setting up the monitor in the online environment.

\section{REFERENCES}

[1] ATLAS Collaboration, ATLAS Technical Proposal, CERN/LHCC/94-43, LHCC/P2, CERN, Geneva, 1994.

[2] ATLAS Collaboration, ATLAS, High Level Triggers, DAQ and DCS Technical Proposal, CERN/LHCC/2000-17, CERN, Geneva, 2000

[3] C. Bee, D. Francis, L. Mapelli, R. McLaren, G. Mornacchi, J. Petersen, F. Wickens, The raw event format in the ATLAS Trigger \& DAQ, ATL-DAQ-98-129, ATL-D-ES-0019, Atlas Communication, CERN, Geneva, 2004

[4] R. Brun, F. Rademakers. (1997). ROOT - An Object Oriented Data Analysis Framework. Nucl. Inst \& Meth. in Phys. Res. A 389, pp. 81 - 86.

[5] ATLAS CTB Website -

http://atlas.web.cern.ch/Atlas/GROUPS/GENERAL/TESTBEAM/ 